الهامش التسويقي لحليب الابقار المنتج محليا في محافظة بغداد قضاء ابي غريب للموسم الانتاجي 2015

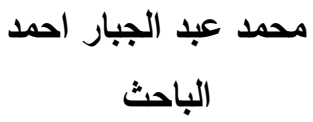

Waneessaeed74@yahoo.com

$$
\text { قسم الاقتصاد الزراعي - كلية الزراعة- جامعة بغداد }
$$

أستاذ مساعد

afafalhani@gmail.com

المستخلص

استهدف البحث حساب الهامش التسويقي كفم واحد من حليب الابقار في محافظة بغداد قضاء ابي غريب، أذ جمعت عينة

قوامها (146) منتج حليب (بقار، و(15) وسيط (جامع حليب) و(21) تاجر تجزئة، اختيرت العينة بطريقة عشوائية، ويلغ فئغ فئن متوسط الهامش التسويقي لكفم حليب ابقار نحو (400) ديناراكفم، موزعة بين تاجر التجزئة والوسيط، اذ بلغ الهامش التسويقي لتاجر التجزئة والوسيط نحو(250، 150) ديناراكفم على الترتيب، اما التكاليف التسويقية لتاجر التجزئة فقد بلغت نحو (81.42، 22.02) ديناراكفم على الترتيب، في حين بلغت ارياح تاجر التجزئة والوسيط نحو(168.58، 168، ديناراكفم على الترتيب، اما نصيب دينار المستهلك المدفوع لكل من المنتج وتاجر التجزئة والوسيط فقد بلغئ(46.7، 20.0\% على الترتيب. ان انخفاض ارياح الوسيط مقارنة بتاجر التجزئة سبيه تكاليف الفاقد والتالف من الحليب نسبة الى لى اجمالي التكاليف التسويقية التي تثكل حوالي (31\%) لذلك يوصي البحث نقل الحليب المبأ بإحكام والمبرد ومنه تقلل نسبة الفاقد والتالف لزيادة ارياح الوسطاء، لدورهم الفعال في عمليات نقل الحليب من المنتج الى المستهلك.

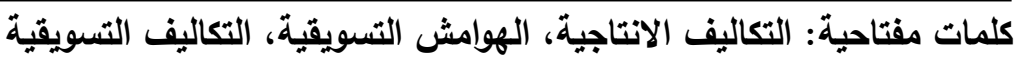
"البحث مستل من رسالة ماجستير للباحث الثاني *

The Iraqi Journal of Agricultural Sciences -618-623: (2) 48/ 2017

HUSSAN \& AHMAD

\title{
MARKETING MARGIN OF LOCALLY PRODUCED MILK COWS IN THE PROVINCE OF BAGHDAD/DISTRICT ABU- GHRAIB FOR PRODUCTIVE SEASON 2015 \\ A.S.HUSSAN \\ Assist. Prof \\ M.A.AHMAD \\ Researcher
}

\section{ABCTRACT}

afafalhani@gmail.com

This research aim to marketing margin account of one $\mathrm{kg}$ milk cows to the province of Baghdad district of Abu Ghraib. Collected a sample of (146) producer cow's milk, (15) broker (milk collector's) and (21) retailer, random sample selection manner. The average marketing margin of one $\mathrm{kg}$ milk cows was about $\mathbf{4 0 0}$ dinars $\backslash \mathrm{kg}$, marketing margin retailer and broker were about $(250,150)$ dinar $\backslash \mathrm{kg}$ respectively, marketing costs of the retailer and broker have amounted to $(81.42,22.02)$ dinar $\backslash \mathrm{kg}$. Retailer and broker profits amounted to about (168.58, 127.98) dinar $\backslash \mathrm{kg}$. Dinar per consumer paid all of the producer, retailer and broker was amounted to $(46.7,33.3,20.0) \%$, respectively. That causing the decline in broker profits compared to the retailer caused by costs and losses spoiled milk, To the total marketing costs Which caculated for approximately $(31 \%)$. The results were recommended to using the transfer of milk packed tightly and refrigerated, reduce waste .

Key words: Production costs, marketing margin, marketing costs

*Part of M.Sc. thesis of the second author. 
اسلوب إنتاج الحليب المتبع لدى اغلب المنتجين(مربي الماشية) يكون بشكل تقليدي، فعمليات البيع نكون اغلبها عن إن التهاج

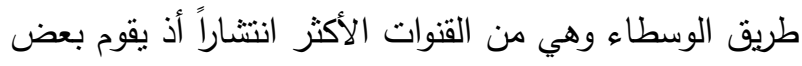
تجار الحليب الوسطاء بتجميعها من المنتجين مباشرة ونقلها

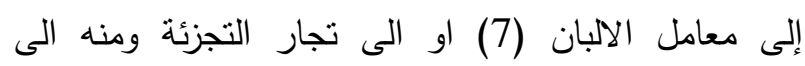
المستهلك، وبعض المنتجين يبيعون الحليب ومنتجاته مباشرة الى تجار التجزئة، أو من خلال الباعة المتجولين الذين يوصلونها للمستهلاك النهائي (2) وغالباً ما يتم النقل بسيارات الثحن الصغيرة (البيك اب)، فالحليب الموزع بهذه الطريقة

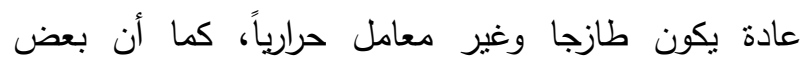
المنتجين يحول الحليب الى منتجات اخرى في منزله بالطرق التقليدية ، لذا فأن الحليب يعاني من مشاكل انتاجية وتسويقية

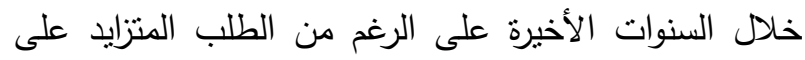

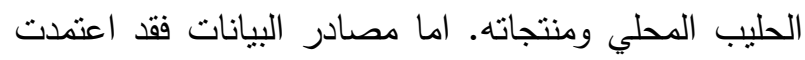
الدراسة أعداد استمارة استبانة لكل من (المنتج، الوسيط، تاجر التجزئة) اذ بلغ اعداد المنتجين في عينة البحث نحو النه

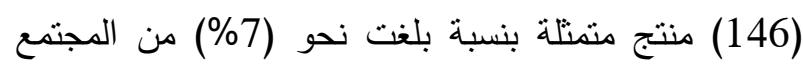

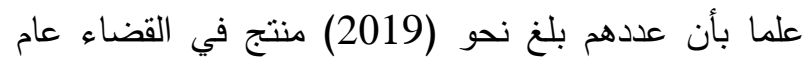
2015، اما الوسطاء وتجار التجزئة فبلغت اعدادهم في عينة البحث نحو(15، 21) على الترتيب، علما بأنه لا نوجد احصائية رسمية نتير الى اعدادهم في المجتمع في شعبة

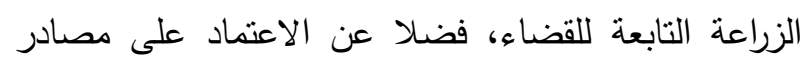

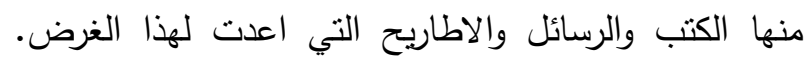
اشار باحثون اخرون (3، 5، 8، 14، 16) الى دراسة هيكلة بنود تكاليف وانتاج الحليب، ومن نتائج بعض الباحثين (1)، 4، 9، 10، 12) اشارت الى دراسة الهيكل التسويقي للحليب الخام، فقد اهتم البحث بقياس الهامش النسويقي وقياس ارباح الاطراف المشاركة في تسويق الحليب ومنهم الوسطاء الذين

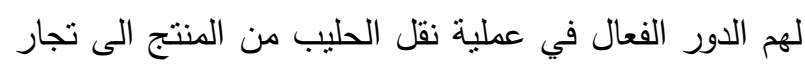

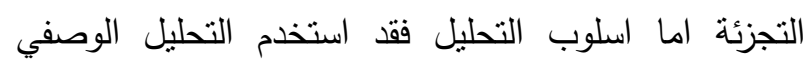

\section{والكمي لهذه الدراسة. \\ المواد وطرائق العمل}

1- لحساب الهامش التسويقي والذي يعرف على انه الفرق بين السعر الذي ينسلمه المنتج والسعر الذي يدفعه المستهلك (18)، استعملت المعادلات الاتية (11):

الهامش التسويقي المطلق= سعر التجزئة - سعر باب الحقل التل
تعد الثروة الحيوانية ذات اهمية اقتصادية أذ بلغت اهميتها النسبية كمعدل للسنوات (2009- 2013) نحو 22\% من قيمة الناتج الزراعي (17) اما الاهمية الاقتصادية للحليب

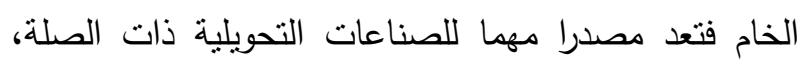
وتعتمد منتجات الالبان بصفة اساسية على الحليب كونه المكون الاساسي للمشاريع المتخصصة في صناعة الالبان والتي احتلت مكانة اقتصادية مهمة، ويسمى الحليب الذي الذي التئي تتتجه الابقار باسم الحليب الخام. اما القيمة الغذائية للحليب

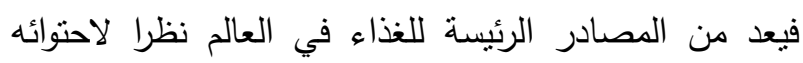

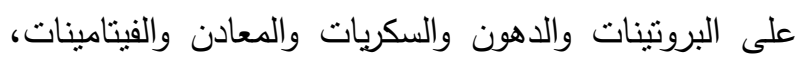

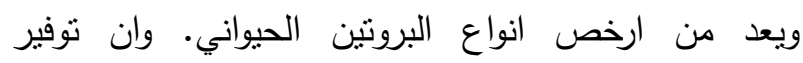
المنتجات الحيوانية بشكل عام والحليب بشكل خاص تواجد النه الكثير من المعوقات بسبب الطلب المتزايد عليها لأسباب عديدة منها زيادة الوعي الثقافي والصحي لدى الافراد والزيادة

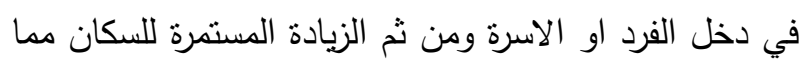

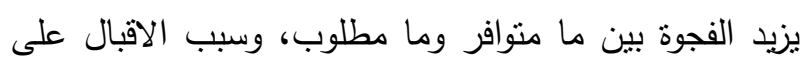

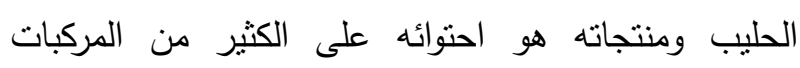
والعناصر الغذائية الضرورية لحياة الانسان وصحته مقارنة بالمواد الغذائية النباتية الاخرى، لذا يتطلب توافر هذه المادة

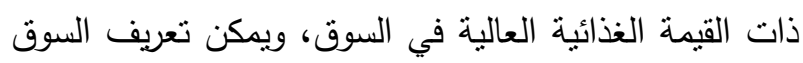

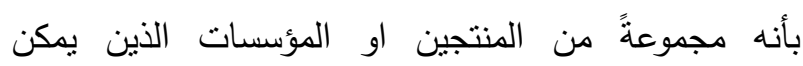
الاستقادة من منتجاتهم او خدماتهم التي يقدموها مع وجود الظروف التنافسية في السوق وفي المقابل وجود المشترين لتلك المنتجات او الخدمات ولديهم القدرة الثرائية والرغبة في وجي الثراء(13). فيما يخص الجانب التسويقي، حصلت تغيرات سلوكية تخص المنتجين في كيفية اختيار افضل الخيارات التسويقية لتسويق الحليب الخام، اذ توفرت عدد من القنوات والمسالك التسويقية تسهم في اختيار المربي الى افضل مسلك تصنيف تسويقي يتبعه، ويتضمن دور الوسطاء الزراعيين بين

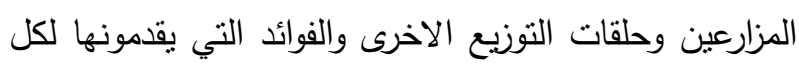
من المنتج والمستهلك سواء بسواء (15)، وان برامج نسويق الحليب نتأثز ايضا بعدد من العوامل المرنبطة بالعملية

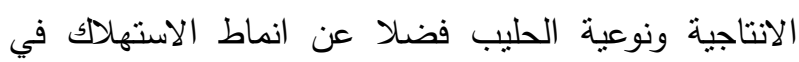
الاسواق المستهدفة والتي تختلف من شريحة لأخرى. تعد الأندائ

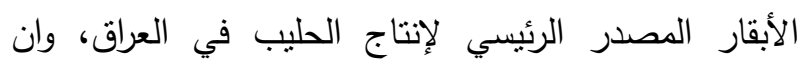


الرأس، وتشكل الحقول الصغيرة من اجمالي عدد الحقول الكلي في منطقة الدراسة نحو 71 \% اما التكاليف الكلية اللازمة لإنتاج كفم حليب فقد قسمت لحت الى :-

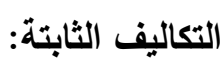
واهمها تكاليف (اهلاك الوحدة الحيوانية، قيمة ايجار الحقل،

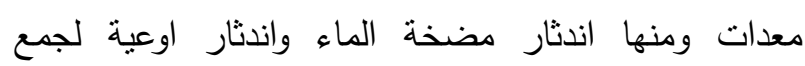

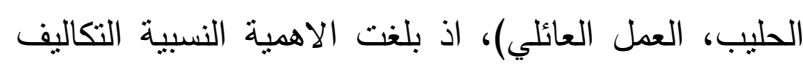

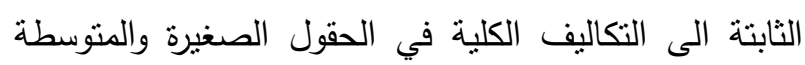
والكبيرة نحو 26.40، 22.28، 22.68\% على التزتيب، كما في جدول 1. وتشمل تكاليف (الاعلاف بأنواعها المركزة والخضراء

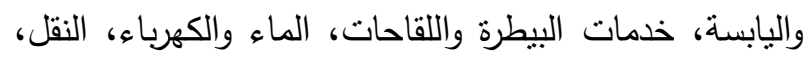
الوقود والصيانة)، اذ بلغت الاهمية النسبية التكاليف المتغيرة الى التكاليف الكلية في الحقول الصغيرة والمتوسطة والكبيرة نحو 73.60، 77.72، 77.32\% على الترتيب، ويلاحظ من خلال جدول 1 ان اجمالي التكاليف الثابتة والمتغيرة كقيمة مطلقة ونسبية كانت الاعلى في الحقول الصغيرة بينما انخفضتا في الحقول المتوسطة والكبيرة على الترتيب، ويمكن فئن تعليل ذلك من خلا مزايا الانتاج الكبير الذي تتخفض فيه التكاليف الانتاج كلما توسعت حقول التربية.
الهامش التسويقي للوسيط= سعر بيع الوسيط - سعر باب الحقل

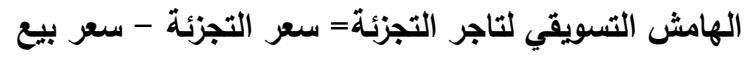
الوسيط

الهامش التسويقي النسبي البيعي= [(سعر التجزئة - سعر المنتج) اسعر التجزئة] * 100 اما التكاليف التسويقية هي التكاليف التي تتفق على الخدمات

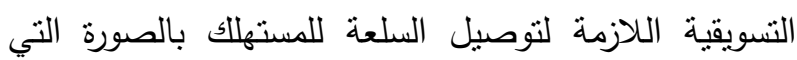

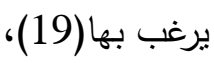

2- توزيع الانصبة التسويقية من خلال الصيغ الاتية: (6)

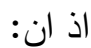

$\mathbf{P s}=(\mathbf{P p} \backslash \mathbf{R p})^{*} \mathbf{1 0 0}$

$\mathrm{Bs}=[(\mathbf{B p}-\mathbf{P p}) \backslash \mathbf{R p}] * \mathbf{1 0 0}$

$\mathbf{R s}=[(\mathbf{R p}-\mathbf{B p}) \backslash \mathbf{R p}] * \mathbf{1 0 0}$

Ps نصيب المنتج، Bs : نصيب الوسيط، Rs : نصيب تاجر التجزئة، Pp: سعر المنتج، Rp سعر التجزئة، Bp: سعر الوسيط. النتائج والمناقشة النشة إن اهم النتائج المتحصل عليها من العينة هي ان اعداد

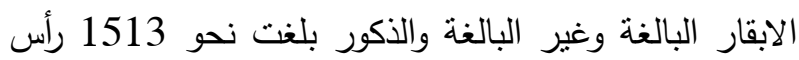

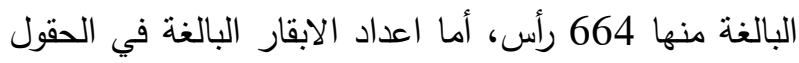
الصغيرة والمنوسطة والكبيرة فبلغت نحو 203، 251، 250، 210 رأس على الترتيب، في حين بلغ متوسط كمية انتاج حليب

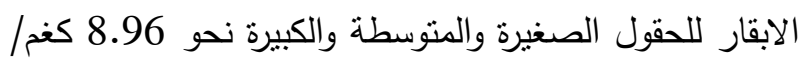

جلول 1. التكاليف الكلية اللازمة لإنتاج كفم حليب الابقار في الحقول الثلاثة مبينا اهميتها النسبية والكلفة مقدرة بالدينار

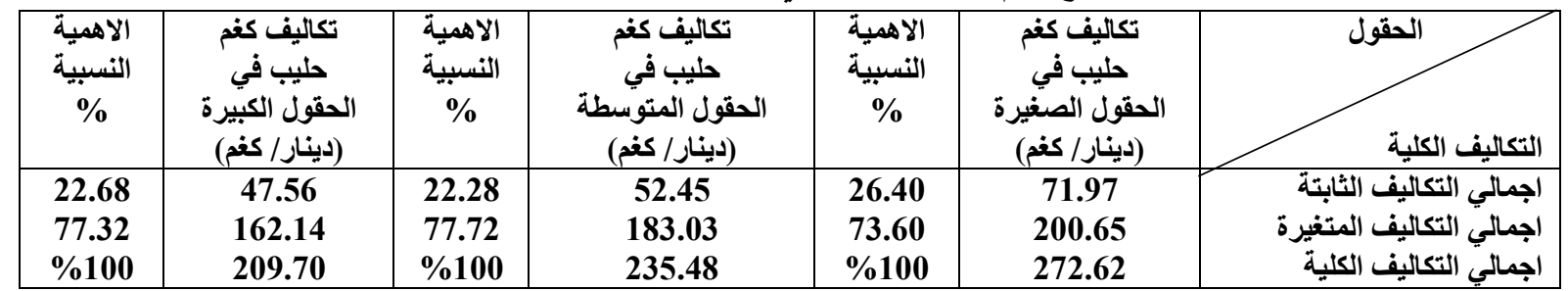

المستهلك عند تاجر التجزئة فبلغ 750 دينار/ كغم، وعليه المصدر: حسبت من قبل الباحثين اعتمادا على استبانة المنتج بلغ منتسط الهامش التسويقي المطلق لكغ حليب ابقار نحو

الهوامش التسويقية 400 دينار / كغ كما في جدول 2. بلغ متوسط سعر كغم حليب ابقار عند باب المزرعة نحو

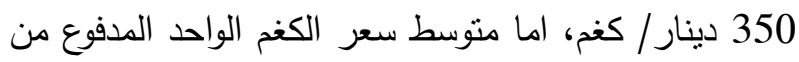
جدول 2 .الهامش التسويقي المطلق والنسبي لكغم حليب الابقار (دينار/ كغم)

\begin{tabular}{|c|c|}
\hline سعر حليب الابقار (دينار/ & البيانات \\
\hline $\begin{array}{c}350 \\
750 \\
400 \\
\% 53.33\end{array}$ & 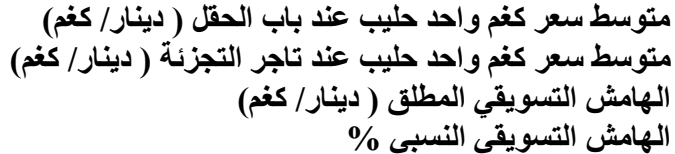 \\
\hline
\end{tabular}

المصدر: حسبت من قبل الباحثين اعتمادا على استبانة المنتج وتاجر التجزئة 
التكاليف التسويقية لكل من المنتجين، الوسطاء، تجار

التجزئة

تختلف التكاليف التسويقية عند مرور الحليب الخام من المنتج وصولا الى ناجر التجزئة في المسلك التسويقي، فالعمليات التسويقية التي يقوم بها منتج الحليب تختلف عن العمليات

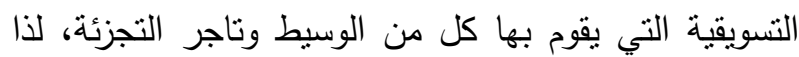

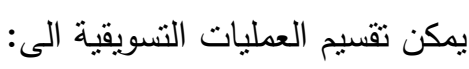

1- العمليات التسويقية للمنتجين لفين من خلال بيانات استمارة الاستبانة تبين ان المنتجين يقومون

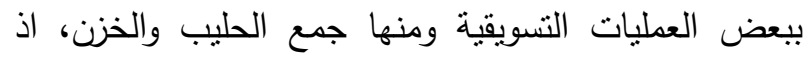
بلغت الاهمية النسبية لجمع الحليب في الحقول الصغيرة والمتوسطة والكبيرة نحو 10.87، 24.41، 33.75\% على الته الترتيب من اجمالي التكاليف التسويقية، اما الخزن اذ بلغت الاهمية النسبية في الحقول الثلاثة نحو 89.13، 75.59، 66.25 على الترتيب من اجمالي التكاليف التسويقية كما

$$
\text { موضحة في جدول } 4 .
$$

ان سعر بيع كغ حليب عند باب المزرعة فبلغ 350 دينار /

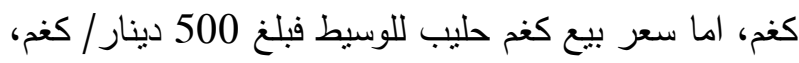
في حين بلغ سعر بيع كغم حليب عند تاجر التجزئة نحو

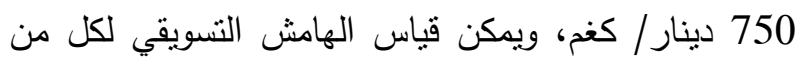
الوسيط وتاجر التجزئة من خلا: ونمن 1- الهامش التسويقي بين سعر الوسيط وسعر المنتج 500- 350= 150 دينار / كغم 2- الهامش التسويقي بين سعر التجزئة وسعر الوسيط=

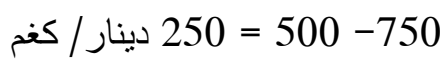
يوضح الجدول 3 الهامش النسويقي لكل من الوسيط وناجر

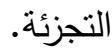

جدول 3 الهامش التسويقي للوسيط وتاجر التجزئة لكفم

\begin{tabular}{|c|c|c|}
\hline \multicolumn{3}{|c|}{ حليب الابقار (دينار/ كغم) } \\
\hline (التسويقي & (دينار/ الفيح) & الاطراف \\
\hline 150 & 500 & الوسيط \\
\hline 250 & 750 & تاجر التجزئة \\
\hline
\end{tabular}

المصدر: حسبت من قبل الباحثين اعتمادا على استبانة الوسيط وتاجر التجزئة

جدول 4 .التكاليف التسويقية لمنتجي حليب الابقار في الحقول الثلاثة (دينار/ كفم)

\begin{tabular}{|c|c|c|c|c|c|c|}
\hline الاهمية & التسويقية التكاليف & $\begin{array}{l}\text { الاهمية } \\
\text { النسبية }\end{array}$ & التي الحقويقية & الاهمية & في التسويقية & \\
\hline 33.75 & 1.60 & 24.41 & 1.56 & 10.87 & 1.65 & الحليب \\
\hline 66.25 & 3.14 & 75.59 & 4.83 & 89.13 & 13.52 & $*$ \\
\hline$\% 100$ & 4.74 & $\% 100$ & 6.39 & $\% 100$ & 15.17 & مالي التكاليف التسويقية \\
\hline
\end{tabular}

اوعية جمع الحليب) اذ بلغت الاهمية النسبية لكل منهم نحو

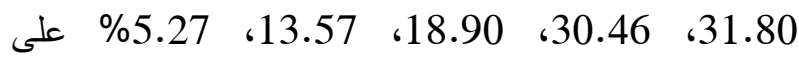
المصدر: حسبت من قبل الباحثين اعتمادا على استمارة استبانة المنتج

التزتيب من اجمالي التكاليف التسويقية كما موضحة في 2- العمليات التسويقية للوسطاء جدول لبيع الحليب الى المستهلك بتكاليف (اجور العمال، ايجار

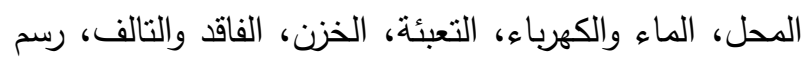
ضريبي) اذ بلغت الاهمية النسبية لكل من اجور العمال

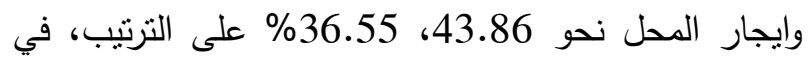
حين بلغت الاهمية النسبية لكل من (الماء والكهرباء، التعبئة، الخزن، الفاقد والتالف، رسم ضريبي) نحو 8.77، 5.84، ك. 5.

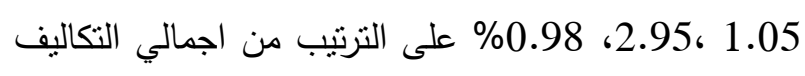

$$
\text { التسويقية كما موضح في جدول } 6 .
$$

\begin{tabular}{|c|c|c|}
\hline النسبيةية الاهبة & تكاليف التعليات & بنود التكاليف \\
\hline 31.80 & 7.0 & الفاقد والتالف \\
\hline 30.46 & 6.71 & نقل الحليب \\
\hline 18.90 & 4.16 & التفريغ والتحميل \\
\hline 13.57 & 2.99 & تبريد الحليب ت \\
\hline 5.27 & 1.16 & اندثار اوعية جمع الحليب \\
\hline$\% 100$ & 22.02 & اجمالي التكاليف \\
\hline
\end{tabular}

جدول 5 .التكاليف التسويقية للوسيط (دينار/ كفم)

المصدر: حسبت من قبل الباحثان اعتمادا على استمارة استبانة الوسيط

شملت العمليات التسويقية التي يقوم بها الوسيط من جمع الحليب من المنتجين ونقل الكمية المسوقة الى تجار التجزئة او معامل الالبان ومنها تكاليف يتحملها الوسيط اهمها (الفاقد والتالف، نقل الحليب، التفريغ والتحميل، تبريد الحليب، اندثار 
3-العمليات التسويقية لتاجر التجزئة

شملت العمليات التسويقية التي يقوم بها تاجر التجزئة

ارياح المنتجين والوسطاء وتجار التجزئة

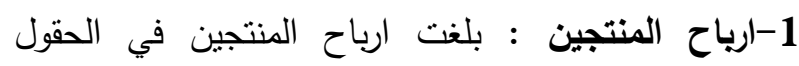
الثناثة نحو 62.21، 108.13،

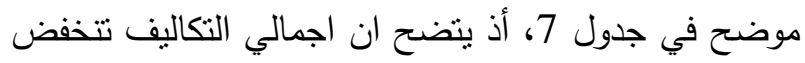
في الحقول الكبيرة في حين تزداد الارباح وفق ميزات الانتاج الكبير
جدول 6. التكاليف التسويقية لتاجر التجزئة (دينار/ كفم)

\begin{tabular}{|c|c|c|}
\hline الالنسبية & تكاليف العمليات & بنود التكاليف \\
\hline 43.86 & 35.71 & العامل الواحـ \\
\hline 36.55 & 29.76 & ايجار المحل \\
\hline 8.77 & 7.14 & الماء و الكهرباء \\
\hline 5.84 & 4.76 & التعبئة \\
\hline 1.05 & 0.86 & 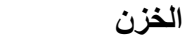 \\
\hline 0.98 & 0.81 & رسم ضريبي \\
\hline 2.95 & 2.38 & الفاق او التالف \\
\hline$\% 100$ & 81.42 & اجمالي التكاليف \\
\hline
\end{tabular}

المصدر: حسبث من قبل الباحثين اعتمادا على استمارة استبانة تاجر

جدول 7. ارياح المنتجين في الحقول الثلاثة (دينار/ كفم)

\begin{tabular}{|c|c|c|c|}
\hline (دينار / كغم) الكبيرة & الحقول المتوسطة / كنم) & (دينار / كفم) الصغيرة & البيانات \\
\hline 214.44 & 241.87 & 287.79 & اجمالي التكاليف الانتاجية والتسويقية \\
\hline 350 & 350 & 350 & متوسط سعر بيع الكغم الواحد \\
\hline 135.56 & 108.13 & 62.21 & صافي الارياح \\
\hline
\end{tabular}

التجزئة تفوق ارباح الوسيط بنسلة اكثر من 40\% لكل كغم

المصدر: حسبت من قبل الباحثين اعتمادا على استبانة المنتج

حليب مباع.

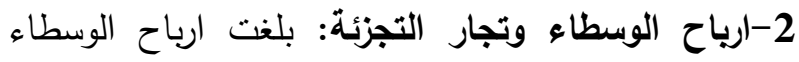

وتجار التجزئة نحو 127.98،

الترنيب كما موضح في جدول 8، اذ يتضح ان ارباح ناجر

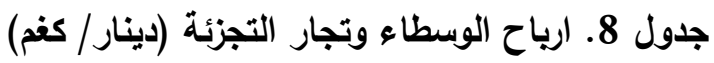

\begin{tabular}{|c|c|c|c|c|c|c|c|}
\hline التباح تاجئة & سعر بيعِ تاجزئة & 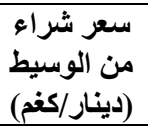 & التكاليف التجزئة التبقية & 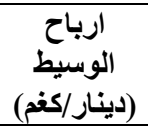 & سلو الوسيط & سن سعر شتراء & التكاليف التسويطية \\
\hline 168.58 & 750 & 500 & 81.42 & 127.98 & 500 & 350 & 22.02 \\
\hline
\end{tabular}

29\% من اجمالي اعداد الحقول في منطقة الدراسة، اما في

المصدر حسبت من قبل الباحثين اعتمادا على استبانة الوسيط وتاجر

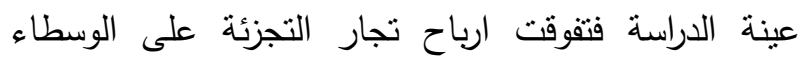

والمنتجين وعلى الرغم من انخفاض التكاليف التسويقية

للوسطاء عن تجار التجزئة الا ان النتائج اظهرت نسبة التهائ خسائر (الفاقد والتالف) للوسطاء اعلى من تجار التجزئة وهذا يدل على عدم اهتمام الوسطاء بنقل الحليب في أوعية محكمة ومبردة بشكل امتل مما نسبب في انخفاض ارباحهم، اما نصيب المنتج والوسيط وتاجر التجزئة من دينار المستهلاك المدفوع فقد استحوذ المنتج على النسبة الاعلى من نصيب مليط الدينار المدفوع من المستهلك وهذا لا يعكس مدى ربحية

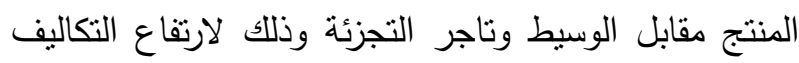

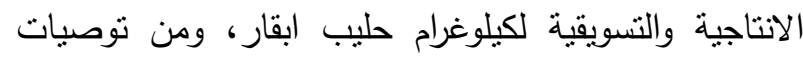
البحث على الوسطاء اخذ دورهم الفعال في نقل الحليب باستخدام تعبئة محكمة ومبردة مما يزيد من ارباحهم وتقليل

توزيع الانصبة التسويقية للمنتج والوسيط وتاجز التجزئة من دينار المستهلك الصنا بلغ نصيب المنتج من دينار المستهلك نحو 46.7 \% وهو

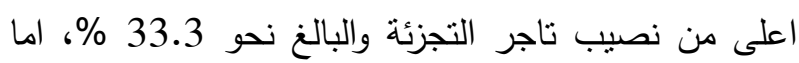
نصيب الوسيط فقد بلغ نحو 30.0 \% كما في جدول 9. جدول 9. توزيع نصيب دينار المستهلك لكغم حليب ابقار

\begin{tabular}{|c|c|c|}
\hline نصيب التجزئة & نصيب الوسيط & نصيب المنتج \\
\hline 33.3 & 20.0 & 46.7 \\
\hline
\end{tabular}

المصدر حسبت من قبل الباحثين اعتمادا على استبانة كل من المنتج والوسيط وتاجر التجزئة اظهرت النتائج في عينة المنتجين، ارتفاع ارباح المنتجين في والجية الحقول الكبيرة والمتوسطة مقارنة بالحقول الصغيرة، الا ان

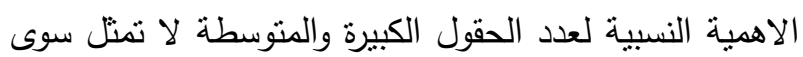




\section{REFERENCES}

1. ALHunaiti D. A. R., 2007. "Revenue and marketing systems for livestock products in the Jordan Badia South" research presented to the Faculty of Agriculture, Muta- University (Jordan), Journal of the University of King Saud, Vol. 19, No. (2), p:123.

2. Ali Y. M, 2000, "Fluid milk", National Library for printing and publishing, Mosul, p:52.

3. ALMiqdad F., 2012, 'An Economic study of The costs of the production of cow's milk in the Countryside of the Province of Damascus', University Damascus of Agric- ultural Sciences Journal, 28, (2), p:393.

4. Alraee M. I. , 2004, "Dairy Industry in Palestine between Reality and Prospects" Working Paper, the General Administration of Studies and Planning, Ministry of National Economy, p: 13.

5. ALTarawneh M. S. and A. Ali, 2011, "Estimate raw milk and margin marketing cost in cattle farms in Jordan", Research Presented to the Department of Agricultural Economics, College of Agriculture, University of Damascus, Damascus University Journal of Agricultural Sciences, (27) (1): 379.

6. Andrew W. Sh., 2007, "A Guide to Marketing Costs and how to Calculate them," Agricultural Management, Marketing and Finance Service Rural Infrastructure and Agro-Industries Division, p: 52.

7. Arab Organization for Agricultural Development, 2003, "The Development of Production, Processing and Marketing of Dairy with small- scale Farmers in the Arab world", Sudan, Khartoum, p: 4.

8. Bipradas R., 2014, "Studies on Marketing Efficiency of Agricultural Products in India: A Critical Evaluation", International Journal of Multidisciplinary Research and Development 1(7), p :205-210 .

9. Chisoni M., 2012, "Economic Analysis of The Viability of Small Holder Dairy Farming in Zambia", M.Sc. Thesis Science in Livestock Economics, The University of Zambia, p: 158.

10. Debnarayan S. and K. Bikash, 2010, "Milk marketing under cooperative and nonCooperative marketing channels: evidence from West Bengal" ,Marketing Efficiency, Economic Analysis, Volume LV, No. 187, October- December.

11. FAO, 2006, "Agricultural and Food Marketing Management" Calculate Marketing Margin, cha. (12), 2 van 24, p: 305.

12.Fikrineh N. E., 2012 "Production, handling, processing, utilization and marketing of milk in the Mid Rift Valley of Ethiopia" lives- tock research for rural Development 24 (9).

13. Michael W. L. 2006, "Market Definition is a Multi-Dimensional Process" BP Trends Jun, www.bptrends.com, p: 2.

14.Dayanandan R., 2011, "Production and marketing efficiency of dairy farms in Highland of Ethiopia- An Economic Analysis", International Journal of Enterprise Computing and Business, Vol. 1 Issue 2 July, p: 230 .

15. Obaidat M., 2000, "Agricultural Marketing", Dar Wael for publication, Jordan, p: 17.

16. Rolf, J. and G. , 2004, "Efficiency Losses in Milk Marketing" Foundation for Research in Economic and Business Administration Bergen, March, p:1503 .

17. The Republic of Iraq, Ministry of Agriculture, the Department of Planning and Follow up, the Department of Statistics Data, for the years $2009-2013$.

18. Yassin M. 2007, "The entrance to the attention of the Agricultural Marketing", Faculty of Agriculture, University of Damascus publications, $\mathrm{p}: 249$.

19. Zouubi A., 2006, "Principles of Agricultural Marketing," Dar Al-Hamed library, the first edition, Amman, Jordan, p: 77. 